\title{
Efeito da antraquinona na curva de cozimento kraft para madeira do híbrido Eucalyptus grandis x Eucalyptus urophylla
}

\author{
Magnos Alan Vivian ${ }^{1 *}$, Francides Gomes da Silva Júnior ${ }^{2}$ \\ 'Universidade Federal de Santa Catarina, Centro de Ciências Rurais, Rod. Ulysses Gaboardi, Km 3, CEP 89520-000, Curitibanos, SC, Brasil \\ ¿Universidade de São Paulo, Escola Superior de Agricultura "Luiz de Queiroz", Av. Pádua Dias, 11, CEP 13418-900, Piracicaba, SP, Brasil
}

\section{"Autor correspondente: \\ magnos.alan@ufsc.br}

Termos para indexação:

Fabricação de pasta celulósica

Aditivo de cozimento

Polpa de madeira

Index terms:

Pulping

Cooking additive

Wood pulp

Histórico do artigo:

Recebido em 17/05/2016

Aprovado em 10/11/2017

Publicado em 29/12/2017

doi: 10.4336/2017.pfb.37.92.1267

\begin{abstract}
Resumo - O presente estudo teve por objetivo avaliar os efeitos da adição da antraquinona em uma curva de cozimento, com diferentes cargas de reagentes, por meio do processo kraft de polpação, para a madeira de híbrido de Eucalyptus grandis $\mathrm{x}$ Eucalyptus urophylla, visando inferir sobre os parâmetros do processo. A madeira foi transformada em cavacos e classificada para condução das curvas de cozimento kraft. As cargas aplicadas foram de $10 \%$ a $24 \%$ de álcali ativo e $25 \%$ de sulfidez, com uma carga de antraquinona de $0,05 \%$ (sobre a massa seca de madeira). Avaliou-se o rendimento bruto e depurado, o teor de rejeitos, o número kappa (nível de deslignificação) e o álcali ativo residual. Observou-se que a adição da antraquinona não afetou o rendimento bruto do processo, porém aumentou o rendimento depurado através da redução do teor de rejeitos, em especial para as baixas cargas de álcali ativo (10\% e 12\%). Outro efeito observado foi a redução do número kappa com a adição da antraquinona, o que indica o aumento da taxa de deslignificação da madeira. Esse aumento é reforçado pelo menor álcali ativo residual, o que se subentende maior aproveitamento dos reagentes no processo.
\end{abstract}

\section{Anthraquinone effect on kraft cooking curve for the wood of Eucalyptus grandis x Eucalyptus urophylla hybrid}

\begin{abstract}
This study aimed to evaluate the effects of anthraquinone addition in the cooking curve, with different loads of reagents, through the kraft pulping process, of Eucalyptus grandis $\mathrm{x}$ Eucalyptus urophylla wood, to infer about the process parameters. The wood was transformed into chips and classified to conduct the kraft cooking curves. The applied loads were from $10 \%$ to $24 \%$ of active alkali, with an anthraquinone charge of $0.05 \%$ (on dry wood). We evaluated raw and screened yield, reject content, kappa number (delignification level) and residual active alkali. We observed that the addition of anthraquinone did not affect the process raw yield, but increased the screened yield by reducing reject content, especially for low loads of active alkali (10\% and $12 \%)$. Another effect observed was the reduction of the kappa number with the addition of anthraquinone, which indicates the increase of the wood delignification rate. This is reinforced by the lower residual active alkali, which implies greater use of reagents in the process.
\end{abstract}

\section{Introdução}

Hoje o Brasil é o líder mundial na produção de celulose de fibra curta, no qual se destaca o uso da madeira de espécies ou híbridos do gênero Eucalyptus, em especial o Eucalyptus grandis x Eucalyptus urophylla. O país é o segundo maior produtor mundial de celulose. Em 2016 o país produziu 18,8 milhões de toneladas de celulose, dos 
quais $86,2 \%$ oriundas de espécies ou híbridos de fibra curta, com um acréscimo de $8,0 \%$ em relação a 2015 para esse tipo de fibra (Indústria Brasileira de Árvores, 2017).

Em produção de celulose, o Brasil só fica atrás dos Estados Unidos, que apresentou em 2016 um volume produzido de 48,5 milhões de toneladas (Indústria Brasileira de Árvores, 2017). Atualmente, grandes empresas brasileiras do setor de celulose e papel estão investindo na duplicação de algumas unidades industriais ou na melhoria das atuais, que possivelmente consolidará o país no posto de segundo maior produtor de celulose do mundo.

Esse crescimento é ocasionado, além de investimentos na estruturação de novas unidades, por pesquisas e novas tecnologias, especialmente para o eucalipto, que possibilitem a melhoria dos processos de produção de celulose, que contribuam para o aumento da eficiência destes, aliando sempre à qualidade da matéria-prima e do produto final.

Um dos pontos que é observado pelas empresas relaciona-se ao uso de aditivos durante o processo de polpação. Um dos aditivos mais conhecidos é a antraquinona (AQ). Tal composto já é empregado industrialmente, e, contribui para a melhoria do rendimento do processo. Entre as principais razões para a aplicação da AQ nos processos industriais estão, segundo Biasca (1998), o aumento de rendimento, menor produção de sólidos no licor negro e redução do número kappa, por meio da aceleração da taxa de deslignificação.

Durante o processo de cozimento kraft com AQ, as principais reações provocadas por este aditivo de processo consistem basicamente na oxidação dos polissacarídeos da madeira e na hidrólise de ligações éteres da lignina (Gomide \& Oliveira, 1979). De acordo com Jerônimo et al. (2000), a oxidação provocada pela AQ no grupo redutor dos polissacarídeos estabiliza-o em relação às reações de despolimerização terminal. Ainda, segundo os mesmos autores, essa estabilização resulta em proteção destes polissacarídeos contra reações de degradação e solubilização, proporcionando, consequentemente, o aumento do rendimento.

Em relação à dosagem do aditivo, diversos estudos já foram desenvolvidos, os quais citam valores em torno de $0,05 \%$ de $\mathrm{AQ}$, em relação à massa seca de madeira, como o mais indicado para espécies de eucalipto. Um desses estudos foi desenvolvido por Carneiro et al. (2003) para madeira de eucalipto, no qual os autores observaram um incremento de rendimento e de viscosidade com aumento da dosagem de AQ, sendo que o maior efeito foi na dosagem entre 0,04 e $0,06 \%$ (base seca). Robles et al. (1997), em estudo desenvolvido com a madeira de Eucalyptus grandis, com adição de $0,05 \%$ de AQ (base seca), observaram aumento do rendimento depurado quando comparado com o cozimento kraft de referência. Os benefícios da adição da AQ variam de acordo com a carga de reagentes aplicada na polpação kraft, sendo necessário analisar esse quesito de forma pontual.

Desta forma, o presente estudo teve por objetivo avaliar os efeitos da adição da antraquinona em uma curva de cozimento, com diferentes cargas de reagentes, por meio do processo kraft de polpação, para a madeira do híbrido de E. grandis $x$ E. urophylla, visando inferir sobre os parâmetros do processo.

\section{Material e métodos}

Foram utilizadas cinco árvores de um clone do híbrido Eucalyptus grandis $x$ Eucalyptus urophylla, com cinco anos de idade, proveniente de um povoamento localizado no município de Telêmaco Borba, PR. De cada árvore retirou-se um torete com $1 \mathrm{~m}$ de comprimento, que foram transformados em uma amostra composta de cavacos em picador laboratorial. Estes cavacos foram classificados em peneiras de barra (espessura), sendo utilizados os que passaram pela peneira de $6 \mathrm{~mm}$, e ficaram retidos na espessura de $4 \mathrm{~mm}$. Na sequência, os cavacos classificados foram submetidos à secagem ao ar e armazenadas em sacos de polietileno.

Para caracterização do material, realizaram-se as análises de densidade básica e aparente dos cavacos e composição química da madeira. A determinação da densidade básica dos cavacos seguiu o método do máximo teor de umidade, conforme descrito pela norma NBR 11941 (Associação Brasileira de Normas Técnicas, 2003), a partir da média aritmética de 10 amostras de $100 \mathrm{~g}$ de cavacos cada (Equação 1).

$$
\mathrm{Db}=\frac{1}{\frac{\mathrm{Mu}}{\mathrm{Ms}}-0,346}
$$

Em que: $\mathrm{Db}$ - densidade básica $\left(\mathrm{g} . \mathrm{cm}^{-3}\right) ; \mathrm{Mu}$ - massa úmida (g); Ms - massa seca (g).

A densidade aparente dos cavacos foi determinada pelo método volumétrico, através da massa seca de cavacos necessária para completar um determinado 
volume. Para determinação do volume utilizou-se um recipiente de $2 \mathrm{~L}$, completando-o com cavacos até a marcação (2 L). Após determinou-se o teor seco dos mesmos para obter-se a massa seca utilizada, a partir da qual calculou-se a densidade aparente (Equação 2), considerando a média de 10 repetições.

$$
\mathrm{Da}=\frac{\mathrm{Ms}}{\mathrm{Va}}
$$

Em que: $\mathrm{Da}$ - densidade aparente $\left(\mathrm{g} . \mathrm{cm}^{-3}\right) ; \mathrm{Ms}$ - massa seca $(\mathrm{g}) ; \mathrm{Va}$ - volume aparente $\left(\mathrm{cm}^{3}\right)$.

Para caracterização química da madeira utilizou-se amostras de cavacos, as quais foram reduzidas à serragem em moinho, classificadas em um conjunto de peneiras com malha de 40 a 60 mesh. Foram determinados os parâmetros: teor de extrativos totais, teor de lignina total e teor de holocelulose (celulose e hemicelulose), conforme metodologia descrita por Vivian (2015). O teor de cinzas foi determinado em mufla, de acordo com as recomendações da norma TAPPI T 211 OM-02 (Technical Association of the Pulp and Paper Industry, 2007). Os resultados obtidos para análise química são expressos como a média de 5 repetições.

\section{Curvas de cozimento}

Os cavacos da madeira de E. grandis x E. urophylla foram submetidos a curvas de cozimento pelo processo de polpação kraft com diferentes níveis de carga alcalina, com e sem adição de antraquinona, em uma proporção de $0,05 \%$ sob massa de madeira (Tabela 1 ). Para realização das curvas de cozimento, utilizou-se autoclave rotativa, com capacidade para $20 \mathrm{~L}$, contendo 8 cápsulas de aço inoxidável individualizadas, com capacidade para $450 \mathrm{~mL}$ cada, realizados em triplicata.

Tabela 1. Condições da curva de cozimento pelo processo de polpação kraft.

\begin{tabular}{cc}
\hline Parâmetros & Condições \\
\hline Álcali ativo, $(\%$ como $\mathrm{NaOH})$ & $10,12,14,16,18,20,22$ e 24 \\
Sulfidez $(\%)$ & 25 \\
Massa seca de cavacos $(\mathrm{g})$ & 70 \\
Relação licor/madeira & $4: 1$ \\
Temperatura máxima $\left({ }^{\circ} \mathrm{C}\right)$ & 166 \\
Tempo de aquecimento $(\mathrm{min})$ & 90 \\
Tempo de cozimento $(\mathrm{min})$ & 60 \\
Fator H & 780 \\
Carga de antraquinona $(\%)$ & 0 e 0,05 \\
\hline
\end{tabular}

Após o processo de polpação, a polpa obtida foi lavada com água até que todo o licor existente fosse visualmente removido. Em seguida, a mesma foi desagregada em "hidrapulper" laboratorial, acondicionada em sacos de microfibras e centrifugada. A depuração da polpa foi realizada em depurador laboratorial com fenda de $0,2 \mathrm{~mm}$; após esta etapa, os rejeitos foram coletados na superfície da tela e secos em estufa a $103 \pm 2{ }^{\circ} \mathrm{C}$. Com os valores do teor de rejeitos secos calculou-se o rendimento depurado. Posteriormente, a polpa celulósica foi centrifugada e armazenada para determinação do número kappa. O licor negro foi recolhido para determinação do álcali ativo residual, seguindo as metodologias descritas na Tabela 2.

Tabela 2. Parâmetros determinados no processo de polpação kraft.

\begin{tabular}{|c|c|}
\hline Parâmetros & Normas/cálculos \\
\hline Rendimento bruto (\%) & $\begin{array}{l}\text { Relação entre massas secas de polpa e de } \\
\text { madeira }\end{array}$ \\
\hline $\begin{array}{l}\text { Rendimento depurado } \\
(\%)\end{array}$ & $\begin{array}{c}\text { Relação entre massas secas de polpa depurada } \\
\text { e de madeira }\end{array}$ \\
\hline Teor de rejeitos $(\%)$ & $\begin{array}{l}\text { Relação entre massas secas de rejeitos } \\
\text { (material retido na peneira com fenda de } 0,2 \\
\text { mm do depurador laboratorial) e de madeira }\end{array}$ \\
\hline Número kappa & $\begin{array}{c}\text { Método TAPPI T } 236 \text { OM-99 (Technical } \\
\text { Association of Pulp and Paper Industry, 2007). }\end{array}$ \\
\hline $\begin{array}{c}\text { Álcali ativo residual } \\
\text { do licor negro, base } \\
\mathrm{NaOH}\end{array}$ & Método descrito por Segura (2012) \\
\hline
\end{tabular}

\section{Resultados e discussão}

Os valores de densidade básica e aparente, assim como a composição química da madeira de Eucalyptus grandis x Eucalyptus urophylla, podem ser observados na Tabela 3.

Tabela 3. Caracterização da madeira de Eucalyptus grandis $x$ Eucalyptus urophylla.

\begin{tabular}{cc}
\hline Parâmetro & Valores médios \\
\hline Densidade básica $\left(\mathrm{g} \mathrm{cm}^{-3}\right)$ & 0,447 \\
Densidade aparente $\left(\mathrm{g} \mathrm{cm}^{-3}\right)$ & 0,167 \\
\hline Composição química & \\
Holocelulose (\%) & 70,25 \\
Lignina (\%) & 27,12 \\
Extrativos (\%) & 2,63 \\
Cinzas (\%) & 0,21 \\
\hline
\end{tabular}

A densidade básica encontrada para a madeira utilizada no presente estudo (Tabela 3) é semelhante aos valores citados por Duarte (2007) e Queiroz \& Gomide (2003), de $0,458 \mathrm{~g} \mathrm{~cm}^{-3}$ e $0,447 \mathrm{~g} \mathrm{~cm}^{-3}$, para árvores 
com 5 e 6 anos de idade, respectivamente. Santos \& Sansígolo (2007), em estudo realizado com a madeira do híbrido de E. grandis x E. urophylla com 6,5 anos de idade, também observaram uma densidade básica média dos cavacos de 0,440 g. $\mathrm{cm}^{-3}$, em conformidade com o presente estudo. No entanto, a densidade da madeira pode variar em função da idade, sítio, espaçamento, bem como do manejo (desbaste e desrama) empregados no povoamento. Por exemplo, Almeida (2003) e Bassa (2007) encontraram densidade um pouco mais alta para cavacos da madeira do híbrido aos 7 anos de idade $\left(0,499 \mathrm{~g} \mathrm{~cm}^{-3}\right.$ e $0,505 \mathrm{~g} \mathrm{~cm}^{-3}$, respectivamente).

Segundo Burger \& Richter (1991), a faixa ideal de densidade para produção de celulose situa-se entre $0,4 \mathrm{~g} \mathrm{~cm}^{-3}$ a $0,6 \mathrm{~g} \mathrm{~cm}^{-3}$, intervalo no qual devem estar às árvores em idade adequada de corte. Alencar (2002), em estudo realizado com a madeira de E. grandis $\mathrm{x}$ E. urophylla de 1 a 7 anos de idade, observou que a densidade básica da madeira se estabiliza com 5 anos. Desta forma, segundo Duarte (2007), isso justifica os estudos realizados com madeiras com idade de 5 e 6 anos. A mesma autora cita ainda que os avanços silviculturais dos plantios de eucalipto no Brasil possibilitam a redução da idade de corte, sendo que algumas empresas brasileiras já estão cortando madeira de eucalipto com 5 anos de idade visando a produção de polpa celulósica.

A densidade aparente observada $\left(0,167 \mathrm{~g} \mathrm{~cm}^{-3}\right)$ foi inferior a valores encontrados na literatura. Longue Júnior \& Colodette (2011), trabalhando com cavacos da madeira do híbrido E. grandis $\mathrm{x}$ E. urophylla com 7 anos de idade, observaram valor médio de $0,193 \mathrm{~g} \mathrm{~cm}^{-3}$ para tal parâmetro. Segura (2012) encontrou o valor médio ainda maior $\left(0,201 \mathrm{~g} \mathrm{~cm}^{-3}\right)$ para a madeira do mesmo híbrido com 6 anos de idade. $\mathrm{O}$ mesmo autor cita que a densidade aparente é um parâmetro simples e direto, que se relaciona com a capacidade de produção de uma fábrica de celulose. Além disso, a densidade aparente também se relaciona com as dimensões dos cavacos, pois a mesma é determinada por meio do método volumétrico. Desta forma, com a classificação se obtém maior homogeneidade nas dimensões, evitando-se os cavacos finos e fora dos padrões ideais.

Os resultados observados para a composição química da madeira de E. grandis x E. urophylla (Tabela 3) estão em consonância com os citados na literatura, entre os quais estão os descritos por Duarte (2007), que caracterizou a madeira de E. grandis $\mathrm{x}$ E. urophylla com idade variando de 5 a 6 anos, observando extrativos variando de $2,30 \%$ a $3,98 \%$, lignina total de $24,53 \%$ a $30,06 \%$, e teor de holocelulose $65,93 \%$ a $72,82 \%$. Segura (2012), trabalhando com a madeira do mesmo híbrido, aferiu que a mesma era composta por 3,06\% de extrativos, $28,09 \%$ de lignina total e $68,85 \%$ de holocelulose. O teor de lignina observado está próximo ao valor apresentado por Queiroz \& Gomide (2003), que utilizaram o mesmo híbrido, e encontraram valor de lignina de $27,20 \%$.

\section{Curvas de cozimento kraft}

Os parâmetros obtidos para a curva de cozimento da madeira de $E$. grandis $\mathrm{x} E$. urophylla através do processo kraft de polpação, com e sem antraquinona (AQ) estão apresentados na Figura 1.

O rendimento bruto (Figura 1a) tende a reduzir com o aumento da carga alcalina aplicada, como já era esperado, pois ocorre maior nível de deslignificação da madeira, assim como maior degradação dos polissacarídeos, pois o licor de cozimento não é totalmente seletivo. A adição da AQ não causou efeito significativo no rendimento bruto, especialmente nas cargas menores de álcali ativo $(\mathrm{AA}=10 \%$ a $14 \%)$. No entanto, as cargas mais altas de AA (a partir de 20\%) acarretaram melhor o rendimento bruto, o que pode ser explicado pela proteção ocasionada pela AQ sobre os polissacarídeos. Conforme Gomide \& Oliveira (1979), a AQ provoca a oxidação dos polissacarídeos da madeira que, de acordo com Jerônimo et al. (2000), os estabiliza em relação às reações de despolimerização terminal, o que resulta em proteção destes polissacarídeos contra reações de degradação e solubilização, proporcionando o aumento do rendimento.

A adição da $A Q$ melhorou o rendimento depurado do processo, reduziu o teor de rejeitos, bem como o número kappa, o que pode ser visualizado nas Figuras 1b, 1c e 1d, em especial para as baixas cargas de álcali. Gomide \& Oliveira (1980), estudando o processo kraft encontraram que a AQ tem ação mais pronunciada em cozimentos com cargas de álcali ativo mais baixas, que de acordo com os autores pode ser explicado pela alta concentração de íons $\mathrm{OH}^{-}$nas cargas mais altas de álcali ativo, o que pode mascarar a ação da AQ.

De acordo com Jerônimo et al. (2000), a intensificação observada na taxa de deslignificação após a adição da AQ se dá através das reações de hidrólise das ligações $ß$-éter, ocasionando a formação de fragmentos de lignina de menor massa molar, os quais são mais facilmente removidos. 

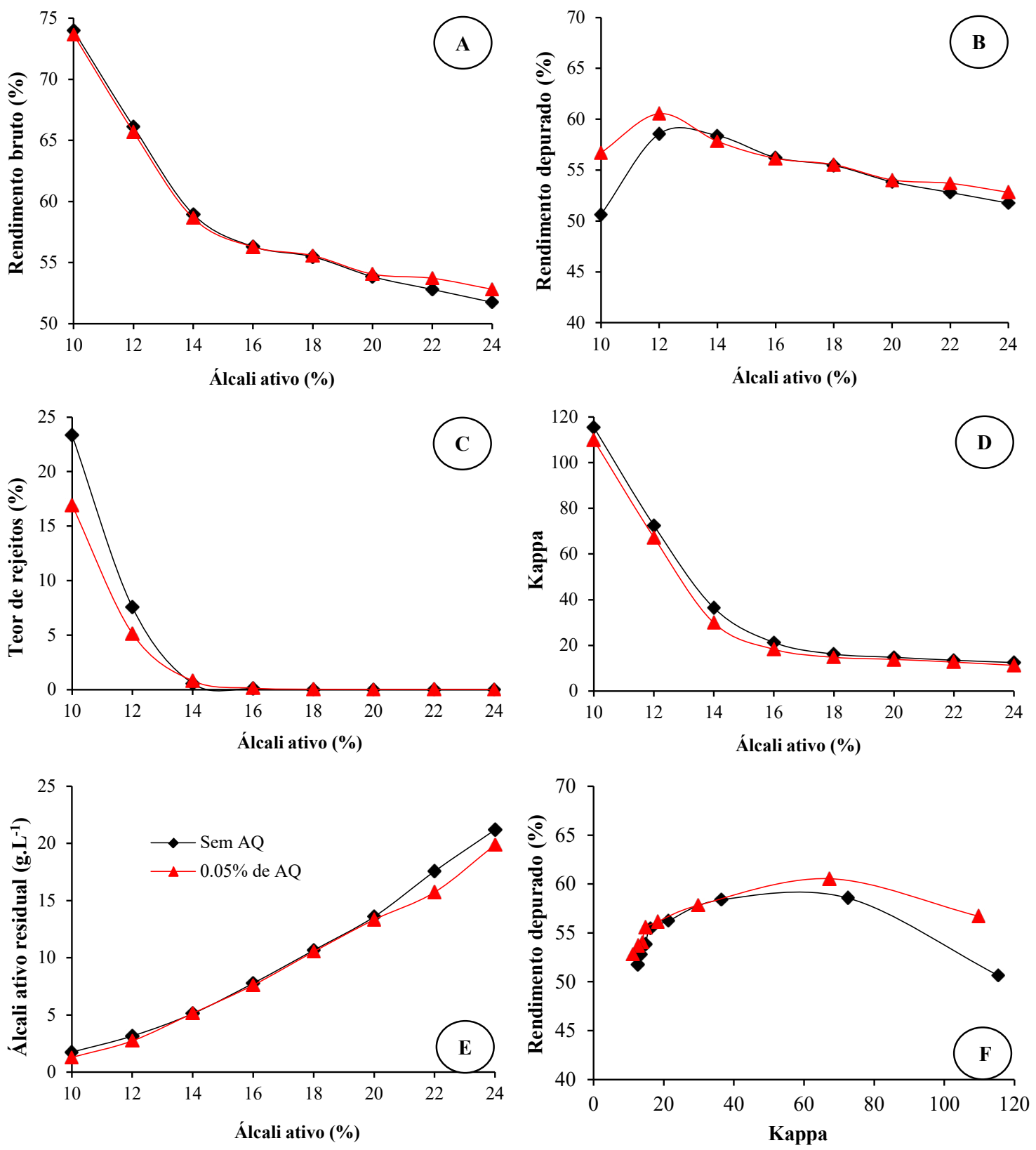

Figura 1. Parâmetros obtidos em função da carga alcalina referente às curvas de cozimento com e sem antraquinona (AQ).

Silva Júnior (1997), em estudo desenvolvido com a madeira de E. grandis x E. urophylla, aplicando diferentes cargas de AQ, observou que a adição da mesma aumenta o rendimento depurado, bem como reduz o teor de rejeitos e número kappa da polpa, indicando que existe um melhor aproveitamento da carga alcalina.
Robles et al. (1997), em estudo desenvolvido com a madeira de $E$. grandis, com adição de $0,05 \%$ de AQ (base seca), observaram aumento do rendimento depurado em todos os níveis de deslignificação estudados quando comparados com os cozimentos kraft de referência. Com base no mesmo estudo, os autores observaram que a polpa kraft-AQ consumiu menos energia para ser 
refinada e apresentou propriedades de resistências iguais ou superiores, especialmente em relação a resistência à tração ao arrebentamento.

Em relação ao nível de deslignificação, expresso pelo número kappa, segue a mesma tendência observada para o rendimento bruto, ou seja, quanto maior a carga de álcali, menor o valor encontrado. Segundo Cardoso et al. (2011), a aplicação de cargas alcalinas mais elevadas fazem aumentar a velocidade de deslignificação (para uma dada temperatura e tempo de reação), obtendose polpas com maior grau de deslignificação, assim, menores valores de número kappa.

A elevação da taxa de deslignificação é acompanhada pelo decréscimo do rendimento causado pela degradação da celulose e hemiceluloses, o que afeta as propriedades de resistência da polpa (Carvalho, 1999). Desta forma, a carga alcalina e a taxa de deslignificação devem ser equilibradas, de acordo com o objetivo final da polpa. Neste contexto, por razões econômicas e de qualidade da polpa celulósica, Cardoso et al. (2011) citam que se deve empregar o mínimo de álcali, visando a obtenção de determinado número kappa. Em contrapartida, Bugajer et al. (1980) fazem o alerta de que cargas de álcali muito baixas podem ocasionar uma queda do $\mathrm{pH}$ do licor durante o cozimento, que pode provocar a reprecipitação de lignina sobre as fibras, ocasionando elevação do número kappa.

Segundo Cardoso et al. (2011), as reações de deslignificação iniciam-se a temperaturas na ordem de $140{ }^{\circ} \mathrm{C}$, sendo as temperaturas mais utilizadas entre $160{ }^{\circ} \mathrm{C}$ e $170{ }^{\circ} \mathrm{C}$. Temperaturas superiores a $175^{\circ} \mathrm{C}$ tornam a celulose mais susceptível à degradação. Segundo os mesmos autores, geralmente, o uso de maior carga de álcali resulta em uma diminuição do rendimento. Tal comportamento ocorre de forma mais evidente em folhosas, de acordo com Bugajer et al. (1980), especialmente pela maior dissolução de xilanas.

Em relação ao álcali ativo residual (AAR) o mesmo tende a ser maior quanto maior a carga alcalina aplicada durante o cozimento (Figura 1e). De acordo com Grace et al. (1989), deve-se procurar manter a concentração de álcali ativo residual do licor na faixa de 5 a 12 g. $\mathrm{L}^{-1}$ (base $\mathrm{NaOH}$ ), a qual corresponde a $\mathrm{pH}$ acima de 12, para evitar a reprecipitação de lignina na polpa. Segundo Silva Júnior (1997), quando o $\mathrm{pH}$ do licor negro atinge patamares inferiores a 11,5 , inicia-se um processo de reprecipitação da lignina sobre a fibra, que é totalmente indesejável, sobretudo em polpas que serão destinadas ao processo de branqueamento. O conhecimento do AAR é importante e justifica-se, pois da carga de álcali ativo aplicada no cozimento, cerca de $2 / 3$ é utilizada para neutralização de ácidos orgânicos, oriundos das reações existentes no processo de polpação, e apenas $1 / 3$ é efetivamente utilizado nas reações de deslignificação (Gomide, 1980).

As cargas de álcali de $10 \%$ e $12 \%$ apresentaram AAR abaixo de 5 g.L $\mathrm{L}^{-1}$ para ambos os processos, podendo provocar a reprecipitação da lignina na polpa. As cargas de $14 \%, 16 \%$ e $18 \%$ apresentaram AAR dentro da faixa, enquanto que as cargas de $20 \%, 22 \%$ e $24 \%$ apresentaram valores superiores ao intervalo citado de AAR.

A adição da antraquinona reduziu a quantidade de álcali ativo residual, o que comprova o melhor aproveitamento dos reagentes durante a polpação, resultando em um maior rendimento e aumento na taxa de deslignificação (menor número kappa), reflexo do melhor aproveitamento da carga aplicada.

A relação entre o rendimento depurado e número kappa (Figura 1f) possibilita uma análise interessante, pois indica qual será o rendimento depurado para um mesmo nível de deslignificação. Para um mesmo número kappa, observa-se que a adição da $A Q$ proporciona maior rendimento depurado em praticamente todos os níveis de kappa.

$\mathrm{Na}$ Tabela 4 podem ser observadas as equações ajustadas a partir dos resultados laboratoriais das curvas de cozimento para os cavacos de referência (sem AQ) e com adição de antraquinona ( $0,05 \%$ de $\mathrm{AQ})$.

Com base nas equações ajustadas, a partir das curvas de cozimento, é possível estimar os parâmetros do processo para polpa, com e sem AQ, em função dos possíveis objetivos a serem alcançados. Foram então simuladas as cargas alcalinas que seriam necessárias para se obter polpa com número kappa 18 , bem como os parâmetros dos processos que seriam obtidos com a aplicação da referida carga (Tabela 5).

Observa-se que a adição de AQ no processo de polpação kraft para obtenção de polpa com número kappa 18 (Tabela 5) reduziria a demanda de AA, aumentaria o rendimento bruto e depurado, assim como melhoraria o aproveitamento dos reagentes aplicados, através da redução do AAR. 
Tabela 4. Equações ajustadas a partir das curvas de cozimento

\begin{tabular}{|c|c|c|c|}
\hline Material & Parâmetro & Equação & $\mathbf{R}^{2}$ \\
\hline \multirow{5}{*}{ Sem AQ } & K & $\mathrm{K}=\frac{1}{\left(0,133288-\frac{1,34528}{\mathrm{AA}}\right)}$ & 0,95 \\
\hline & $\mathrm{RB}$ & $\mathrm{RB}=\frac{1}{\left(0,0235981-\frac{0,0991366}{\mathrm{AA}}\right)}$ & 0,97 \\
\hline & $\mathrm{RD}$ & $\mathrm{RD}=56,9994-0,007431 \times \mathrm{AA}^{2}$ & 0,11 \\
\hline & TR & $\mathrm{TR}=\left(-4,15184+\frac{81,9511}{\mathrm{AA}}\right)^{2}$ & 0,85 \\
\hline & AAR & $\mathrm{AAR}=-2,75089+0,0414679 \times \mathrm{AA}^{2}$ & 0,99 \\
\hline \multirow{5}{*}{$0,05 \%$ de $\mathrm{AQ}$} & K & $\mathrm{K}=\frac{1}{\left(0,145286-\frac{1,45635}{\mathrm{AA}}\right)}$ & 0,96 \\
\hline & $\mathrm{RB}$ & $\mathrm{RB}=\frac{1}{\left(0,0230518-\frac{0,0916692}{\mathrm{AA}}\right)}$ & 0,96 \\
\hline & $\mathrm{RD}$ & $\mathrm{RD}=59,9236-0,0129391 \times \mathrm{AA}^{2}$ & 0,73 \\
\hline & TR & $\mathrm{TR}=\left(-3,48231+\frac{69,7737}{\mathrm{AA}}\right)^{2}$ & 0,88 \\
\hline & AAR & $\mathrm{AAR}=-2,52915+0,0389112 \times \mathrm{AA}^{2}$ & 0,98 \\
\hline
\end{tabular}

Em que: $\mathrm{AQ}$ - antraquinona; $\mathrm{K}$ - número kappa; $\mathrm{RB}$ - rendimento bruto (\%); $\mathrm{RD}$ - rendimento depurado (\%); TR - teor de rejeitos (\%); AA - álcali ativo aplicado (\%); AAR - álcali ativo residual $\left(\mathrm{g} \mathrm{L}^{-1}\right)$.

Tabela 5. Parâmetros estimados a partir das equações para polpa com número kappa 18.

\begin{tabular}{cccccc}
\hline $\begin{array}{c}\text { Material / } \\
\text { Parâmetro }\end{array}$ & $\begin{array}{c}\text { AA } \\
(\%)\end{array}$ & $\begin{array}{c}\text { RB } \\
(\mathbf{\%})\end{array}$ & $\begin{array}{c}\text { RD } \\
(\mathbf{\%})\end{array}$ & $\begin{array}{c}\text { TR } \\
(\mathbf{\%})\end{array}$ & $\begin{array}{c}\text { AAR } \\
\left(\mathbf{g ~ L}^{-1}\right)\end{array}$ \\
\hline Sem AQ & 17,3 & 56,0 & 54,8 & 0,3 & 9,7 \\
$0,05 \%$ de AQ & 16,3 & 57,4 & 56,5 & 0,7 & 7,7 \\
\hline
\end{tabular}

Em que: $\mathrm{AQ}$ - antraquinona; $\mathrm{AA}$ - álcali ativo aplicado (\%); $\mathrm{RB}$ - rendimento bruto (\%); RD - rendimento depurado (\%); TR - teor de rejeitos (\%); AAR - álcali ativo residual $\left(\mathrm{g} \mathrm{L}^{-1}\right)$.

\section{Conclusões}

A adição da antraquinona (AQ) nas curvas de cozimento kraft não afeta o rendimento bruto do processo. No entanto, aumenta o rendimento depurado e reduz o teor de rejeitos para as baixas cargas de álcali ativo.

Além disso, a adição de AQ intensifica a taxa de deslignificação da polpa (observada pela redução do número kappa) e melhora o aproveitamento do álcali ativo aplicado (observado através do menor álcali ativo residual).

Desta forma, o uso da AQ reduz a demanda de reagentes necessários no processo de polpação, através do melhor aproveitamento destes, melhorando a taxa de deslignificação.

\section{Referências}

Alencar, G. S. B. Estudo da qualidade da madeira para produção de polpa celulósica relacionada à precocidade na seleção de um Híbrido Eucalyptus grandis x Eucalyptus urophylla. 2002. $73 \mathrm{f}$. Dissertação (Mestrado em Recursos Florestais) - Escola Superior de Agricultura "Luiz de Queiroz", Universidade de São Paulo, Piracicaba.

Almeida, F. S. Influência da carga alcalina no processo de polpação Lo-Solids ${ }^{\circledR}$ para madeiras de eucalipto. 2003. $115 \mathrm{f}$. Dissertação (Mestrado em Recursos Florestais) - Escola Superior de Agricultura "Luiz de Queiroz", Universidade de São Paulo, Piracicaba.

Associação Brasileira de Normas Técnicas. NBR 11941: madeira: determinação da densidade básica. Rio de Janeiro, 2003. 6 p.

Bassa, A. G. M. C. et al. Misturas de madeira de Eucalyptus grandis x Eucalyptus urophylla e Pinus taeda para produção de celulose kraft através do Processo Lo-Solids ${ }^{\circledR}$. Scientia Forestalis, n. 75, p. 19-29, 2007.

Biasca, K. L. A survey of pulp mill use of anthraquinone. Tappi Journal, v. 81, n. 1, p. 78-79, 1998.

Bugajer, S. et al. Otimização do processo de polpação kraft de eucalipto. O Papel, São Paulo, n. 2, p. 41-47, 1980.

Burger, L. M. \& Richter, H. G. Anatomia da madeira. São Paulo: Nobel, 1991. 154 p.

Cardoso, G. V. et al. Efeito do teor de lignina da madeira de Eucalyptus globulus Labill. no desempenho da polpação kraft. Ciência Florestal, v. 21, n. 1, p. 133-147, 2011. DOI: 10.5902/198050982756.

Carneiro, G. J. C. et al. Aumento de rendimento através do uso de antraquinona os resultados da Bahia Sul Celulose. In: SEMINÁRIO SOBRE PRODUÇÃO DE CELULOSE - NOVAS PRÁTICAS E TECNOLOGIA, 2003, São Paulo. Anais... São Paulo: ABTCP, 2003.

Carvalho, M. G. V. S. Efeito das variáveis de cozimento nas características químicas de pastas kraft de Eucalyptus globulus. 1999. 269 f. Tese (Doutorado em Engenharia Química) Universidade de Coimbra, Coimbra.

Duarte, F. A. S. Avaliação da madeira de Betula pendula, Eucalyptus globulus e de híbrido de Eucalyptus grandis $\mathbf{x}$ Eucalyptus urophylla destinadas à produção de polpa celulósica kraft. 2007. 107 f. Dissertação (Mestrado em Recursos Florestais) - Escola Superior de Agricultura "Luiz de Queiroz", Universidade de São Paulo, Piracicaba.

Gomide, J. L. Antraquinona: Um eficiente aditivo para polpação alcalina de madeiras. Viçosa, 1980. 27 p. (Boletim técnico, 6). 
Gomide, J. L. \& Oliveira, R. C. Eficiência da antraquinona na polpação alcalina do eucalipto. O Papel, v. 41, n. 1, p. 67-72, 1980.

Gomide, J. L. \& Oliveira, R. C. Eficiência da antraquinona na polpação alcalina de eucalipto. Revista Árvore, v. 3, n. 2, p. 208220, 1979.

Grace, T. M. et al. Pulp and paper manufacture: volume 1: Alkaline pulping. Atlanta: TAPPI, 1989. p. 15-22.

Indústria Brasileira de Árvores. IBÁ: Indústria Brasileira de Árvores. Brasília, DF, 2017. 80 p. Relatório IBÁ. Disponível em: < http:// iba.org/images/shared/Biblioteca/IBA_RelatorioAnual2017.pdf $>$. Acesso em: 18 dez. 2017.

Jerônimo, L. H. et al. Adição de antraquinona na polpação alcalina de Eucalyptus saligna. Ciência Florestal, v. 10, n. 2, p. 31-37, 2000. DOI: $10.5902 / 19805098468$.

Longue Júnior, D. \& Colodette, J. L. Remoção de hemiceluloses da madeira por tratamento de auto-hidrólise. Ciência Florestal, v. 21, n. 3, p. 541-550, 2011. DOI: 10.5902/198050983811.

Queiroz, S. C. S. \& Gomide J. L. Efeito das características anatômicas e químicas na densidade básica da madeira de clones de Eucalyptus grandis x Eucalyptus urophylla. O Papel, v. 64, n. 5, p. 79-84, 2003.
Robles, Y. A. M. et al. Utilização de antraquinona e polissulfeto como aditivos do processo kraft para produção de celulose de Eucalyptus. O Papel, maio, p. 43-52, 1997.

Santos, S. R. \& Sansígolo, C. A. Influência da densidade básica da madeira de clones de Eucalyptus grandis x Eucalyptus urophylla na qualidade da polpa branqueada. Ciência Florestal, v.17, n.1, p. 53-63, 2007. DOI: 10.5902/198050981935.

Segura, T. E. S. Avaliação das madeiras de Eucalyptus grandis x Eucalyptus urophylla e Acacia mearnsii para a produção de celulose kraft pelos processos convencional e Lo-Solids ${ }^{\circledR} .2012$. 99 f. Dissertação (Mestrado em Recursos Florestais) - Escola Superior de Agricultura "Luiz de Queiroz", Universidade de São Paulo, Piracicaba.

Silva Júnior, F. G. Polpação kraft do eucalipto com adição de Antraquinona, polissulfetos e surfactante. 1997. $116 \mathrm{f}$. Tese (Doutorado em Engenharia Química) - Universidade Estadual de Campinas, Campinas.

Technical Association of the Pulp and Paper Industry (Atlanta, EUA). Test methods. Atlanta, 2007. $2 \mathrm{v}$.

Vivian, M. A. Aumento da eficiência do processo kraft de polpação a partir de pré-tratamento de cavacos de madeira de eucalipto. 2015. 125 f. Tese (Doutorado em Recursos Florestais) - Escola Superior de Agricultura "Luiz de Queiroz", Universidade de São Paulo, Piracicaba. 